TI 2013-051/II

Tinbergen Institute Discussion Paper
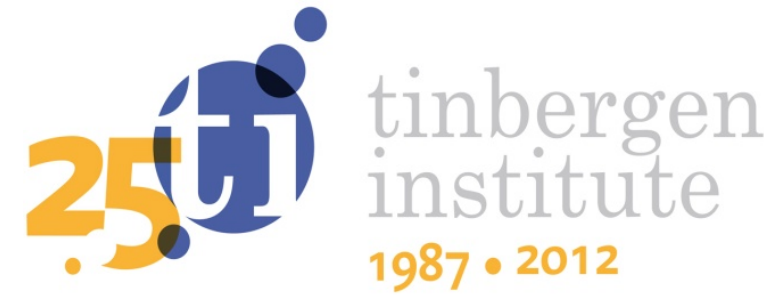

\title{
Asymmetric Nash Solutions in the River Sharing Problem
}

\author{
Harold Houba \\ Gerard van der Laan \\ Yuyu Zeng
}

Faculty of Economics and Business Adminstration, VU University Amsterdam, and Tinbergen Institute. 
Tinbergen Institute is the graduate school and research institute in economics of Erasmus University Rotterdam, the University of Amsterdam and VU University Amsterdam.

More TI discussion papers can be downloaded at http://www.tinbergen.nl

Tinbergen Institute has two locations:

Tinbergen Institute Amsterdam

Gustav Mahlerplein 117

1082 MS Amsterdam

The Netherlands

Tel.: +31(0)205251600

Tinbergen Institute Rotterdam

Burg. Oudlaan 50

3062 PA Rotterdam

The Netherlands

Tel.: +31(0)10 4088900

Fax: $+31(0) 104089031$

Duisenberg school of finance is a collaboration of the Dutch financial sector and universities, with the ambition to support innovative research and offer top quality academic education in core areas of finance.

DSF research papers can be downloaded at: http://www.dsf.nl/

Duisenberg school of finance

Gustav Mahlerplein 117

1082 MS Amsterdam

The Netherlands

Tel.: +31(0)20 5258579 


\title{
Asymmetric Nash Solutions in the River Sharing Problem ${ }^{1}$
}

\author{
Harold Houba ${ }^{2}$ Gerard van der Laan ${ }^{3} \quad$ Yuyu Zeng ${ }^{4}$
}

March 26, 2013

\footnotetext{
${ }^{1}$ This research is financially supported by Netherlands Organization for Scientific Research NWO grant 022.003.035. The authors thank Arantza Estévez-Fernández, Marco Mariotti, Randolph Sloof and the participants of the seminar at Tinbergen Institute for valuable suggestions.

${ }^{2}$ H.E.D. Houba, Department of Econometrics and Tinbergen Institute, VU University Amsterdam, De Boelelaan 1105, 1081 HV Amsterdam, the Netherlands. E-mail: harold.houba@vu.nl

${ }^{3}$ G. van der Laan, Department of Econometrics and Tinbergen Institute, VU University Amsterdam, De Boelelaan 1105, 1081 HV Amsterdam, the Netherlands. E-mail: g.vander.laan@vu.nl

${ }^{4}$ Y. Zeng, Department of Econometrics and Tinbergen Institute, VU University Amsterdam, De Boelelaan 1105, 1081 HV Amsterdam, the Netherlands. E-mail: y.zeng@vu.nl
} 


\begin{abstract}
We study multiple agents along a general river structure that is expressed by a geography matrix and who have access to limited local resources, quasi-linear preferences over water and money and cost functions dependent upon river inflow and own extraction. Unanimity bargaining determines the water allocation and monetary transfers. We translate International Water Law into either disagreement outcomes or individual aspiration levels. In the former case, we apply the asymmetric Nash bargaining solution, in the latter case the agents have to compromise in order to agree and we apply the asymmetric Nash rationing solution. In both cases the optimization problem is separable into two subproblems: the efficient water allocation that maximizes utilitarian welfare given the geography matrix; and the determination of the monetary transfers associated with the weights. We show that the Nash rationing solution may result in nonparticipation, therefore we generalize to the case with participation constraints.
\end{abstract}

Keywords: River Basin Management, International Water Law, Negotiations, Externalities, Political Economy of Property Rights

JEL codes: C70, D60, Q53 


\section{Introduction}

Water is essential. People use it for a variety of purposes: from residential use to industrial production. Due to population growth and industrialization, water demand and pollution levels have tremendously increased and the international community has recognized that fresh water is scarce. Most rivers are transboundary and often agents in upstream locations use too much water and produce pollution. Hence, efficient use and resource management of water serves as an important tool to mitigate fresh water scarcity. While many countries do coordinate their water uses, international disputes do occur. Unfortunately, these disputes cannot be resolved by International Water Law because in essence it only states that the countries involved should mutually agree on sharing the river through negotiations, and it is left in the middle how to resolve disputes over the allocation of water and reduction of pollution. Therefore, some particular characteristics of the river sharing problem such as the externalities of pollution from upstream to downstream and the absence of clearly defined property rights in international river situations have drawn interests from researchers to study the river sharing problem.

Giannias and Lekakis (1996) and Kilgour and Dinar (2001) analyze the river sharing problem between two or more countries along an international river that is linear, i.e., a river originating from one spring where agents are located subsequently from upstream to downstream. The model in the first reference distinguishes between upstream and downstream, is deterministic and has both water quantity and water quality. The second reference analyzes a stochastic model of water quantity among several countries. Both studies characterize the unique allocation that maximizes utilitarian welfare. Ambec and Sprumont (2002) mark the start of embedding legal principles from International Water Law in the river sharing problem. They translate the legal principles of Absolute Territorial Sovereignty (hereafter, ATS) and Unlimited Territorial Integrity (hereafter, UTI) into their model. ATS is applied to every group of agents and can be seen as Core stability. UTI is also applied at the group level, but it is an aspiration approach. It is impossible that distinct groups of countries can simultaneously achieve their group aspiration levels. As appropriate requirements, Ambec and Sprumont (2002) propose group ATS and that no group attains a welfare above its group aspiration level. For a linear river with insatiable agents, they show that the downstream incremental solution is the only welfare distribution that satisfies these two requirements. Ambec and Ehlers (2008) generalize this result by allowing for agents with a fixed satiation point. In van den Brink, van der Laan and Moes (2012), more general river geographies and other legal principles are considered.

International Water Law states that countries should mutually agree on sharing the river through negotiations. For that reason, we approach the river sharing problem from a bargaining perspective. The downstream incremental solution can be interpreted as the outcome of a sequential bargaining process in which the countries along the linear river enter one by one from upstream to downstream and at each entrance the joining member 
gets its incremental contribution. This is as if at every entrance the joining member has the absolute bargaining power to extract all surplus from cooperation, which is not very convincing. In a different context, this is as when new countries join the EU, these countries have the bargaining power to receive all the welfare surplus generated by enlarging the EU. Clearly, there is a need for a more realistic bargaining perspective that allows for less extreme distribution of surpluses.

The literature on bargaining does provide a general theory based upon arbitrary distributions of bargaining power. Because International Water Law states that countries should mutually agree on the water allocation, unanimity among these countries is required. This makes the asymmetric Nash bargaining solution (hereafter, ANBS) a natural candidate for analyzing the river sharing problem. The ANBS has been axiomatized in e.g., Kalai (1977), Kaneko (1980) and Herrero (1989), and it is supported by strategic bargaining models in e.g., Herrero (1989), Miyakawa (2006), Laruelle and Valenciano (2008) and Herings and Predtetchinski (2010). Application of the ANBS to the river sharing problem with only two agents, an upstream and a downstream agent, can be found in e.g. Houba (2008) and Houba, Do and Zhu (2013). This paper generalizes this approach to a general river geography with multiple agents.

As noticed in Houba (2008), legal principles not only restrict the negotiations to unanimity bargaining, but also have implications for the countries' strategic possibilities as long as they do not cooperate, i.e., the disagreement outcome. In this paper we apply the ATS principle and the UTI principle as the guiding principles for individual countries in case of disagreement. For UTI, we discuss two interpretations: a strict interpretation in which only the most downstream country is allowed to use water; and an interpretation in which each country claims UTI. The ATS and strict UTI imply different disagreement outcomes that are both feasible. Under the second UTI interpretation the vector of individual aspiration levels under disagreement is infeasible and yields a utopia point, agreement can only be reached if the countries are willing to compromise on these levels. In Mariotti and Villar (2005) the Nash rationing solution is given and axiomatized to study compromise situations in which unanimity is required. Their solution is symmetric, possibly multi-valued and always contains the maximizers of a modified Nash product over the Pareto frontier. For situations with transferable utility, the Nash rationing solution is unique and coincides with the unique maximizer. In this paper, we propose a modification of the Nash rationing solution to allow for asymmetries.

Compared to the current literature, this paper makes several contributions. First, we model multiple agents along a general river structure that is expressed by a geography matrix and who have access to limited local resources. Each agent has quasi-linear preferences over water and money; where the use of water yields a net benefit being the difference of the benefit of water use and the cost of water extraction. The extraction costs depend upon the amount of the available water and the amount of extraction. This may result in a satiation 
point that depends on the amount of water.

Second, translating the ATS principle and two interpretations of the UTI principle into individual levels of welfare under disagreement, we arrive at three different cases. In the first two cases the vectors of disagreement levels are feasible and serve as the different disagreement outcomes in the ANBS. Different legal principles define different property rights, and therefore the associated ANBS outcomes will also be different and allow an interpretation in terms of a shift in property rights. We also quantify the negotiated welfare distribution. The most downstream agent always prefers the strict UTI principle, because it gives him the right to claim all water inflows along the river. At least one of the other agents prefers the ATS principle, but strong bargainers amongst these agents may prefer the strict UTI principle as well, which differs from the two-agents case.

Since the individual aspiration levels are infeasible, we apply to this situation the asymmetric Nash rationing solution (hereafter, ANRS). The asymmetric weights in this solution cannot be interpreted as bargaining weights, because a larger weight yields a lower welfare. Instead, it is intuitive to interpret these weights as responsibility weights. Mathematically the ANRS has many similarities with the ANBS, but we show by means of an example that at the ANRS some agents might receive a welfare that is below what could be obtained by blocking agreement, i.e., refrain to use water and nonparticipation in the negotiations. To avoid such outcomes we propose to add participation constraints to the asymmetric Nash rationing problem. These constraints can also be justified by modelling a ratification process that takes place after the negotiations are concluded.

Third, we show that the maximization of the (modified) Nash products is separable into two subproblems: the efficient water allocation that maximizes utilitarian welfare and that can be related to the geography matrix; and the monetary transfer associated with the bargaining weight. In order to derive general formulas that are also applicable if the consequences of other legal principles from International Water Law are studied, we analyze the ANBS, respectively ANRS, under unspecified disagreement (utopia) points.

This paper is organized as follows. In Section 2, we specify the river sharing model and introduce the general river geography. Then, in Section 3, we discuss several legal principles and translate them in either a disagreement point or a utopia point. In Section 4, we first analyze the ANBS for unspecified disagreement points and derive the decomposition, before analyzing specific disagreement points associated with the mentioned legal principles. In Section 5 the individual aspiration levels are analyzed from a Nash rationing perspective. Section 6 contains two numerical examples and Section 7 concludes this paper.

\section{Model specification}

We consider a river that flows through a finite set of locations, for instance cities, agriculture communities, industrial facilities or countries, at which water is extracted from the river. 
These locations are called agents and the set of agents is denoted by $N=\{1,2, \ldots, n\}$, where $n \geq 2$ is the number of agents. The river geography is represented by a graph with the agents as its nodes. In this paper we consider directed trees, where the root of the tree represents the most downstream agent, numbered by $n$, and arcs are directed to the root. This collection of possible river geographies includes the linear river in Ambec and Sprumont (2002) and rivers that originate at multiple springs, which merge together downwards into a single stream, as considered in e.g. van den Brink et al. (2012) and Ansink and Houba (2012). Every agent located downstream to agent $i$ is said to be a successor of $i$ and we denote the set of all successors of $i$ by $S^{i}$. Because $n$ is the root of the tree, we have $S^{n}=\emptyset$ and $S^{i} \neq \emptyset$ for all $i \neq n$. Similarly, the set $P^{i}$ denotes the set of all predecessors of $i$ located upstream of $i$ along the river. An agent $i$ has $P^{i}=\emptyset$ if and only if $i$ is located at a spring or source of the river. Furthermore, we notice that $P^{n}=N \backslash\{n\}$.

The natural water inflow, possibly zero, at the territory of agent $i, i \in N$, is denoted by $e_{i}$ and the amount of water used by agent $i$ is denoted by $x_{i}$. Furthermore, all predecessors of agent $i$ could potentially transfer water to $i$, whereas $i$ could possibly transfer water to his successors. The amount of water available for agent $i$ is given by $f_{i}=e_{i}+\sum_{j \in P^{i}}\left(e_{j}-x_{j}\right)$, which consists of his own local water resource $e_{i}$ plus the inflow of water that his predecessors transfer to $i$. Since water only flows from upstream to downstream and inflow at successors of $i$ can not be allocated to $i$, the water use of agent $i$ is constrained by $x_{i} \leq f_{i}$. In the sequel we denote $e=\left(e_{1}, \ldots, e_{n}\right)^{\top} \in \mathbb{R}_{+}^{n}$ as the vector of natural inflows, $x=\left(x_{1}, \ldots, x_{n}\right)^{\top} \in \mathbb{R}_{+}^{N}$ as the vector of water uses and $f=\left(f_{1}, \ldots, f_{n}\right)^{\top} \in \mathbb{R}_{+}^{n}$ as the vector of constraints.

Because it might be convenient to work in matrix notation, following Ansink and Houba (2012) we model the river geography by the $n \times n$ matrix $R$ with components $R_{j i}$ given by $R_{j i}=1$ if $j \in S^{i} \cup\{i\}$, and $R_{j i}=0$ otherwise. Using this allows us to rewrite the vector $f$ of available water and all water constraints $x \leq f$ as

$$
f=e+(R-I)(e-x), \quad \text { respectively, } \quad R x \leq R e .
$$

The next example illustrates the notation.

Example 2.1. Consider a river with two springs at locations 1 and 2 that merge together at location 3. Then the river flows through location 4. Then the matrix representing the river geography is given by

$$
R=\left[\begin{array}{llll}
1 & 0 & 0 & 0 \\
0 & 1 & 0 & 0 \\
1 & 1 & 1 & 0 \\
1 & 1 & 1 & 1
\end{array}\right]
$$

For instance, since water from location 1 can be used in locations 1, 3 and 4, the first column 
of $R$ is given by $\left(\begin{array}{llll}1 & 0 & 1 & 1\end{array}\right)^{\top}$. Then the (in)qualities for water inflows and constraints given by

$$
f=e+(R-I)(e-x) \text { and } R x \leq R e .
$$

indeed yield the (in)equalities

$$
\left[\begin{array}{l}
f_{1}=e_{1} \\
f_{2}=e_{2} \\
f_{3}=e_{3}+\left(e_{2}-x_{2}\right)+\left(e_{1}-x_{1}\right) \\
f_{4}=e_{4}+\left(e_{3}-x_{3}\right)+\left(e_{2}-x_{2}\right)+\left(e_{1}-x_{1}\right)
\end{array}\right] \text { and }\left[\begin{array}{l}
x_{1} \leq e_{1} \\
x_{2} \leq e_{2} \\
x_{1}+x_{2}+x_{3} \leq e_{1}+e_{2}+e_{3} \\
x_{1}+x_{2}+x_{3}+x_{4} \leq e_{1}+e_{2}+e_{3}+e_{4}
\end{array}\right] \text {. }
$$

By the tree structure of the river geography we have the following two properties on the matrix $R$.

\section{Property 2.2.}

(i) If $R_{j i}=1$, then $R_{i j}=0$.

(ii) If $R_{j i}=1$ and $R_{k i}=1$, then either $R_{k j}=1$ or $R_{j k}=1$.

The first property reflects that if water can flow from agent $i$ to $j \neq i$, then it is impossible that the water flows from $j$ to $i$. This rules out locations that have a local common pool, for instance situations in which the river is the common border between two countries. The second property reflects that we don't allow that the river splits into a delta, i.e., each agent $i \neq n$ has precisely one downstream neighbor. This is ruled out, because otherwise additional information is needed regarding how the water flow divides amongst different branches, which may depend on geographical factors, for instance the differences in altitude along the several branches, as well as on the flow level at the point of splitting.

Given the constraints $R x \leq R e$ on the use of water, each agent along the river chooses an amount $x_{i}$ of water use for industrial production, residential use, irrigation etc. An amount $x_{i}$ yields benefits of the water use and costs of water extraction for each agent. Agent $i$ 's cost depends upon the amount of water extraction $x_{i}$ and the available water $f_{i}$.

Assumption 2.3. Agent $i \in N$ has a benefit function $b_{i}: \mathbb{R}_{+} \rightarrow \mathbb{R}_{+}$with the property that $b_{i}^{\prime}>0, b_{i}^{\prime \prime}<0$ and $b_{i}(0)=0$.

Assumption 2.4. Agent $i \in N$ has a cost function $c_{i}: \mathbb{R}_{+}^{2} \rightarrow \mathbb{R}_{+}$with the property that $\frac{\partial c_{i}}{\partial f_{i}}<0, \frac{\partial c_{i}}{\partial x_{i}}>0, \frac{\partial^{2} c_{i}}{\partial x_{i}^{2}}>0$, and $c_{i}\left(f_{i}, 0\right)=0$ for all $f_{i} \geq 0$.

The inequality $\frac{\partial c_{i}}{\partial f_{i}}<0$ means that water use of upstream agents generates negative externalities for downstream agents. The costs of extraction are decreasing in the amount of available water, i.e., more use of water by the predecessors of agent $i$ and thus a decrease of $f_{i}$ results in higher extraction cost for the same amount $x_{i}$. So a decrease in $f_{i}$ yields an upward 
shift of the entire cost function, except at $x_{i}=0$. The conditions on the first and second derivative of $c_{i}$ to the extraction $x_{i}$ of water imply that the cost function is convex in agent $i$ 's own water use. Finally, we assume that zero extraction yields zero costs, independent of $f_{i}$. This assumption is merely made for convenience in Section 5 where it implies zero utility from inaction or nonparticipation in an agreement. None of our other results depends upon our assumption that zero extraction yields zero costs.

We further assume that utility is transferable in the sense that agents are able to transfer utility to each other by making monetary transfers. The monetary transfer to agent $i$ is equal to $t_{i} \in \mathbb{R}$. A positive transfer $t_{i}>0$ means that agent $i$ receives money, $t_{i}<0$ means that agent $i$ has to pay $\left|t_{i}\right|$. A monetary transfer scheme is a vector $t=\left(t_{1}, \ldots, t_{n}\right) \in \mathbb{R}^{n}$ such that there is no financial deficit: $\sum_{i=1}^{n} t_{i} \leq 0$.

The utility of agent $i$ depends on $x_{i}, f_{i}$ and $t_{i}$ and is given by the quasi-linear utility function

$$
u_{i}\left(f_{i}, x_{i}, t_{i}\right)=b_{i}\left(x_{i}\right)-c_{i}\left(f_{i}, x_{i}\right)+t_{i},
$$

where $b_{i}\left(x_{i}\right)-c_{i}\left(f_{i}, x_{i}\right)$ is the net benefit of the water use $x_{i}$ at $f_{i}$. Notice that by our assumptions the net benefit $b_{i}(0)-c_{i}\left(f_{i}, 0\right)$ of inaction is equal to zero for every $f_{i} \geq 0$. Further, notice that the first-order condition

$$
\frac{\partial b_{i}}{\partial x_{i}}-\frac{\partial c_{i}}{\partial x_{i}}=0
$$

might have a solution depending on $f_{i}$, say $s_{i}\left(f_{i}\right)$. In case there is such a solution, $s_{i}\left(f_{i}\right)$ is the satiation point of $i$ that depends on $f_{i}$.

In summary, the river sharing model is fully represented by the quadruple $(N, R, u, e)$, where $N$ denotes the set of agents, $R$ is the river geography, $u$ is the collection of utility functions $\left\{u_{i}\right\}_{i \in N}$ and $e$ is the vector of local water resources. In the remainder of the paper we assume that each agent in this model is a rational utility maximizer and that all benefit functions, cost functions and water resources are common knowledge.

At this stage, we introduce the maximal utilitarian welfare that we denote as $w$. Formally,

$$
\begin{gathered}
w=\max _{x, f \geq 0} \sum_{i=1}^{n} u_{i}\left(f_{i}, x_{i}, 0\right)=\max _{x, f \geq 0} \sum_{i=1}^{n}\left(b_{i}\left(x_{i}\right)-c_{i}\left(f_{i}, x_{i}\right)\right) \\
\text { s.t. } R x \leq R e \text { and } f=e+(R-I)(e-x) .
\end{gathered}
$$

Since individual utilities are transferable through the monetary transfers, the utility possibility set is given by $U=\left\{u \in \mathbb{R}^{n} \mid \sum_{i=1}^{n} u_{i} \leq w\right\}$, see e.g. page 325 of Mas-Colell, Whinston, and Green (1995). Therefore, the maximal utilitarian welfare describes what can be achieved in the river sharing problem $(N, R, u, e)$ in terms of welfare. In the rest of this paper we 
take it for granted that (1) admits a unique maximizer, which we denote by $\left(x^{U W}, f^{U W}\right){ }^{1}$ It might be that $x_{i}^{U W}=0$ for all $i<n$, i.e., welfare is maximized at zero extraction by all agents $1, \ldots, n-1$. However this seems to be unrealistic in practice and also for ease of analysis we exclude this case.

Assumption 2.5. For at least one agent $i=1, \ldots, n-1$, it holds that $x_{i}^{U W}>0$.

Our framework captures some of the influential models of the river sharing problem. Ambec and Ehlers (2008) assume that the (net) benefit function only depends on $x_{i}$, is strictly concave and might have a satiation point $s_{i}$. Under our assumptions, the concavity of the benefit function $b_{i}$ and the convexity of the cost function $c_{i}$ in $x_{i}$ yield a concave net benefit function in $x_{i}$ that might have a satiation point. Since the cost function $c_{i}$ also depends on the available water resources $f_{i}$, also the satiation point $s_{i}\left(f_{i}\right)$ depends upon $f_{i}$, and so our model generalizes Ambec and Ehlers (2008).

Our model can also be interpreted in terms of pollution externalities. For instance, van der Laan and Moes (2012) incorporate pollution in the benefit function and the cost function. In their model, an agent's cost function depends on accumulated own pollution and the pollution of all his upstream agents. If we treat water use as positively correlated with pollution and, for some convex function $\hat{c}_{i}: \mathbb{R}_{+} \rightarrow \mathbb{R}_{+}$define the cost function $c_{i}\left(f_{i}, x_{i}\right)=$ $\hat{c}_{i}\left(f_{i}-x_{i}\right)$, then $c_{i}$ depends upon accumulated water use $\sum_{j \in P^{i} \cup\{i\}} x_{j}$, which is identical as in van der Laan and Moes (2012). Our cost function generalizes from this by allowing for asymmetric effects between upstream pollution and own pollution, but makes the additional assumption that the costs are zero when the own pollution is zero.

\section{$3 \quad$ Legal principles defining property rights}

Legal principles from International Water Law have spurred a new emerging literature in the river sharing problem following Ambec and Sprumont (2002). In this section, we first discuss several legal principles that define different property rights regimes for international rivers, and then, translate these into our framework. As e.g. McCaffrey (2001) writes, two doctrines are prevalent in the International Water Law: the principle of Absolute Territorial Sovereignty (hereafter, ATS) and the principle of Unlimited Territorial Integrity (hereafter, UTI). Each of these principles will be discussed in a separate subsection.

\subsection{Absolute Territorial Sovereignty}

In 1895, the Mexican government filed a complaint against the US government about the excessive use of water from the river Rio Grande, which originates in the US and forms part of

\footnotetext{
${ }^{1}$ Notice that (1) may admit multiple maximizers to support the unique global maximum $w$. Since the uniqueness of $w$ drives our analysis, we assume uniqueness of the maximizer for ease of discussion and to relieve the notational burden.
} 
the border between the US and Mexico before flowing into the Gulf of Mexico. The decision from the Attorney General of the US Department of Justice, Judson Harmon, is that "the United States is under no obligation to Mexico to refrain its use of the Rio Grande because its absolute sovereignty within its own territory entitles it to dispose of the water within that territory in any way it wishes, regardless of the consequences in Mexico" (McCaffrey, 1996). The US therefore had no obligations towards Mexico. In accordance to this, the ATS principle, also known as the Harmon doctrine, states that a country has absolute sovereignty over the area of any river basin on its territory: it may freely decide how much water to use of the water flowing within its borders but cannot claim the continued and uninterrupted flow from upper basin countries.

In our framework, every agent $i$ has the property rights over his own local water resource and inflow from upstream under ATS. In this situation, agent $i$ can freely consume $f_{i}$. This includes his own local inflow $e_{i}$ and all the unused water from his predecessors without the obligation to pay any monetary compensation. Starting from the agents $i$ with $P^{i}=\emptyset$, we can recursively solve for the inflows $f_{i}^{A T S}$ that will result when all agents maximize their own net benefits by

$$
f_{i}^{A T S}=e_{i}+\sum_{j \in P^{i}}\left(e_{j}-x_{j}^{A T S}\right), \quad \text { where } x_{i}^{A T S}=\arg \max _{x_{i}} u_{i}\left(f_{i}^{A T S}, x_{i}, 0\right) \text {, s.t. } x_{i} \leq f_{i}^{A T S} .
$$

The disagreement pair $\left(x^{A T S}, f^{A T S}\right)$ differs from the unique maximizer $\left(x^{U W}, f^{U W}\right)$ of $(1)$, because the ATS does not internalize the externalities of $f_{i}$ on the cost of extraction. So, there exists a group of at least two agents who can beneficially trade water to increase utilitarian welfare.

In negotiations, the net benefits associated with these water uses and inflows specify the disagreement utilities given by $d_{i}^{A T S}=u_{i}\left(f_{i}^{A T S}, x_{i}^{A T S}, 0\right), i=1, \ldots, n$. Since every agent can guarantee himself a zero net benefit by a zero extraction of water, it holds that $d_{i}^{A T S} \geq 0$ for all $i$. The following result shows that the disagreement point under the ATS principle yields strictly less welfare than the maximal utilitarian welfare $w$ as defined in (1). The proofs of the results in this paper are deferred to the appendix.

Proposition 3.1. In the river model $(N, R, u, e)$ it holds that $\sum_{i=1}^{n} d_{i}^{A T S}<w$.

\subsection{Unlimited Territorial Integrity}

Egyptian reliance on the Nile River over the millennia has led it to believe that it has natural and historical rights over those waters. This belief is reflected in the position taken by Egypt in international fora that "no country has the right to undertake any positive or negative measure that could have an impact on the river's flow in other countries", for instance in the Egypt Country Report at the 1981 Interregional Meeting of International River in Dakar. Hence, Egypt is particularly sensitive to any action towards the Nile river by the upstream 
countries and strongly opposed the 2010 agreement on sharing the Nile water signed in Entebbe by Ethiopia, Rwanda, Tanzania and Uganda.

The UTI principle states that a country has the right to demand the natural flow of an international river into its territory that is undiminished in quantity and unchanged in quality by the upstream countries. Incorporating it into our framework, an upstream agent is only allowed to consume water if he has the explicit consent of all his downstream agents. As recognized in e.g. McCaffrey (1996) and (2001), when all agents invoke the UTI principle, UTI itself becomes self-contradictory. In the case of one upstream and one downstream agent, when both agents invoke the UTI principle, the local water resource on the territory of the upstream agent is claimed by both, leading to inconsistency. In the following discussion, we consider two interpretations of the UTI principle: according to the first strict interpretation, only the most downstream agent may claim all water inflows, in the second interpretation the UTI principle is invoked by all agents.

\subsubsection{Strict UTI}

The UTI principle clearly favors downstream agents over upstream agents. Hence, in practice, the UTI principle has often been invoked by downstream agents. In this subsection, we take the most restrictive case under the UTI principle, namely that only the most downstream agent may claim all the water of the river and can restrict all his predecessors to zero extraction as long as no agreement has been reached.

Formally the disagreement utilities are obtained as follows. For every agent $i=1,2, \ldots, n-$ 1 we have that under disagreement $x_{i}^{U T I}=0$ and thus the disagreement flows are given by $f_{i}^{U T I}=e_{i}+\sum_{j \in P^{i}} e_{j}$. For the most downstream agent $n$, we have that $f_{n}^{U T I}=\sum_{j \in N} e_{j}$ and $x_{n}^{U T I}$ is the solution to the the maximization problem

$$
x_{n}^{U T I}=\arg \max _{x_{n}} b_{n}\left(x_{n}\right)-c_{n}\left(f_{n}^{U T I}, x_{n}\right) \text {, s.t. } x_{n} \leq f_{n}^{U T I} .
$$

This gives the disagreement utilities $d_{i}^{U T I}=u_{i}\left(f_{i}^{U T I}, 0,0\right)=0$ for agents $i=1, \ldots, n-1$ and $d_{n}^{U T I}=u_{n}\left(f_{n}^{U T I}, x_{n}^{U T I}, 0\right)=u_{n}\left(\sum_{j \in N} e_{j}, x_{n}^{U T I}, 0\right)>0$. The disagreement utilities under the strict UTI principle yield strictly less welfare than the maximal utilitarian welfare $w$. We state the following result without proof, ${ }^{2}$ and the strict inequality is implied by Assumption 2.5 that $\left(x^{U W}, f^{U W}\right)$ and $\left(x^{U T I}, f^{U T I}\right)$ are unequal.

Proposition 3.2. In the river model $(N, R, u, e)$ it holds that $\sum_{i=1}^{n} d_{i}^{U T I}<w$.

Since $\sum_{i=1}^{n-1} d_{i}^{A T S} \geq 0=\sum_{i=1}^{n-1} d_{i}^{U T I}$ and $d_{n}^{A T S}<d_{n}^{U T I}$, agent $n$ has a strict better bargaining position under UTI than under ATS and the other agents has a reverse order with respect to the bargaining position. However, the final utility also depends on the net

\footnotetext{
${ }^{2}$ The proof is similar to the proof of Proposition 3.1.
} 
surplus (i.e., the welfare from cooperation minus the sum of disagreement utilities). In the next section, we will clarify how these two effects determine the final utility for each agent.

Under strict UTI it might happen that $x_{n}^{U T I}<f_{n}^{U T I}$, so $x_{n}^{U T I}=s_{n}\left(f_{n}^{U T I}\right)=s_{n}\left(\sum_{j \in N} e_{j}\right)$, i.e., agent $n$ extracts his satiation level of water and leaves some of the total flow $\sum_{j \in N} e_{j}$ unused. This raises the issue whether agent $n$ 's predecessors should be allowed to use water. The answer is negative. An intuitive explanation is that any water use by the upstream agents generates negative externalities on the most downstream agent in the sense of decreasing his water inflow, hence increasing his extraction cost. Indeed, denoting $\hat{x}_{n}$ as the solution of $\max _{x_{n} \leq f_{n}^{U T I}-x_{-n}} b_{n}\left(x_{n}\right)-c_{n}\left(f_{n}^{U T I}-x_{-n}, x_{n}\right)$, where $x_{-n}$ denotes the aggregate water use amount by the predecessors of agent $n$, it follows that

$$
\begin{aligned}
& b_{n}\left(\hat{x}_{n}\right)-c_{n}\left(f_{n}^{U T I}-x_{-n}, \hat{x}_{n}\right)<b_{n}\left(\hat{x}_{n}\right)-c_{n}\left(f_{n}^{U T I}, \hat{x}_{n}\right) \leq \\
& \max _{x_{n} \leq f_{n}^{U T I}} b_{n}\left(x_{n}\right)-c_{n}\left(f_{n}^{U T I}, x_{n}\right)=b_{n}\left(x_{n}^{U T I}\right)-c_{n}\left(f_{n}^{U T I}, x_{n}^{U T I}\right),
\end{aligned}
$$

and the strict inequality comes from the fact that $c_{n}\left(f_{n}, x_{n}\right)$ decreases when the water inflow $f_{n}$ increases. Therefore, as long as no agreement has been reached, the most downstream agent will invoke his property rights on all the water resources and forbids his predecessors to consume any water.

\subsubsection{Individual aspiration levels}

Ambec and Sprumont (2002) define agent's $i$ individual aspiration level as the maximal welfare that $i$ would be able to achieve in the absence of all other agents, i.e., when agent $i$ would be able to use the entire water inflow $f_{i}^{U T I}$ at his own territory and the territories of all his upstream agents. ${ }^{3}$ As noticed before, the individual aspiration levels are infeasible when the river contains at least two agents and the agents have to compromise on their aspiration levels in order to reach agreement. Despite feasibility, individual aspiration levels often provide important reference points for individual decision makers. Also, Locke (1948) (see page 24) wrote: "Now, of those good things which nature hath provided in common, every one had a right, as hath been said, to as much as he could use, and property in all he could effect with his labor; all that his industry could extend to, to alter from the state nature had put it in, was his." In this tradition, each agent has a legitimate right to the individual aspiration level, but not to more.

Formally, when all agents invoke the UTI principle, the individual aspiration levels are obtained as follows. For every agent $i$, the inflow under disagreement $f_{i}^{A S P}$ coincides with $f_{i}^{U T I}$, i.e., $f_{i}^{A S P}=e_{i}+\sum_{j \in P^{i}} e_{j}$, and the individual aspiration water use of agent $i$ is given

\footnotetext{
${ }^{3}$ Note that Ambec and Sprumont (2002) also define group aspiration levels for coalitions of agents, which does not appear in our analysis.
} 
by

$$
x_{i}^{A S P}=\arg \max _{x_{i}} b_{i}\left(x_{i}\right)-c_{i}\left(f_{i}^{A S P}, x_{i}\right) \text {, s.t. } x_{i} \leq f_{i}^{A S P} .
$$

This gives the aspiration level utilities $d_{i}^{A S P}=b_{i}\left(x_{i}^{A S P}\right)-c_{i}\left(f_{i}^{A S P}, x_{i}^{A S P}\right) \geq 0$ for all agents $i \in N$. Notice that for all agents $i$ located at a source of the river, i.e., $P^{i}=\emptyset$, we have $d_{i}^{A S P}=d_{i}^{A T S}$ and for the most downstream agent $n$ we have $d_{n}^{A S P}=d_{n}^{U T I}$. The following result extends the infeasibility of individual aspiration levels in Ambec and Sprumont (2002).

Proposition 3.3. In the river model $(N, R, u, e)$ it holds that $\sum_{i=1}^{n} d_{i}^{A S P}>w$.

\section{The ANBS in the river sharing problem}

Agents can improve on the inefficient disagreement outcomes associated with the principles of ATS, respectively strict UTI, by negotiations for joint river management. In this section, we apply the ANBS to mimic such negotiations. This modeling choice can be justified by referring to the 1997 UN Convention that requires consent by all countries in the river basin. It is widely accepted that the ANBS captures unanimity bargaining.

We first establish the ANBS for a general river problem $(N, R, u, e)$ with some maximum welfare $w$ and an unspecified vector $d$ of disagreement utilities with $\sum_{i \in N} d_{i}<w$. Next, we show that by the quasi-linear utilities of the agents the problem to find the ANBS can be decomposed into two smaller subproblems that facilitates its computation: first the utilitarian welfare maximum is computed yielding the efficient water use and second the monetary transfers according to the ANBS are computed. This gives a closed-form solution for the transfers. Finally, we discuss the political economy of property rights by analyzing the ANBS for disagreement utilities associated to the principles of ATS and strict UTI.

\subsection{The bargaining solution}

The ANBS assumes an asymmetric distribution of bargaining weights among $N$ agents. Without loss of generality, these weights are given by a vector $\alpha=\left(\alpha_{1}, \ldots, \alpha_{n}\right)$, where $\alpha_{i} \geq$ 0 and $\sum_{i \in N} \alpha_{i}=1$. In this subsection we further assume the disagreement utilities as exogenously given and impose that every agent $i$ has a disagreement utility $d_{i} \geq 0$ and that $\sum_{i \in N} d_{i}<w$, where $w$ is the maximum welfare that the agents can obtain in the river model $(N, R, u, e)$. The nonnegativity condition is natural given that inaction gives zero utility. Given $w$ and the vector $d$, the bargaining set consists of all utility vectors $u \in \mathbb{R}^{n}$ that are individual rational, thus $u_{i} \geq d_{i}$ for all $i$, and feasible, thus the sum of components is at most equal to $w$.

The ANBS seeks to maximize the asymmetric Nash product $\prod_{i=1}^{n}\left(u_{i}\left(f_{i}, x_{i}, t_{i}\right)-d_{i}\right)^{\alpha_{i}}$ under the constraints that the vector of water uses $x \in \mathbb{R}_{+}^{n}$, the vector of inflows $f \in \mathbb{R}_{+}^{n}$ 
and the vector of monetary transfers $t \in \mathbb{R}^{n}$ are feasible. This gives the following problem ${ }^{4}$

$$
\left.\begin{array}{rl}
\max _{x, f \geq 0 ; t} \quad \prod_{i=1}^{n}\left(b_{i}\left(x_{i}\right)-c_{i}\left(f_{i}, x_{i}\right)+t_{i}-d_{i}\right)^{\alpha_{i}} \text { s.t. } f=e+(R-I)(e-x) \\
& R x \leq R e, \quad(p) \quad \text { and } \sum_{i=1}^{n} t_{i} \leq 0, \quad(\lambda)
\end{array}\right\}
$$

where $p \in \mathbb{R}^{n}$ and $\lambda$ are the Lagrange multipliers for the water resource constraints and monetary transfers, respectively. We have the following result. ${ }^{5}$

Theorem 4.1. Let $x^{*}, f^{*}=e+(R-I)\left(e-x^{*}\right)$ and $t^{*}$ be the water allocation, the vector of inflows and the monetary transfers in the ANBS for the river sharing problem $(N, R, u, e)$. Then $x^{*}$ and $f^{*}$ satisfy the first-order conditions

$$
\begin{aligned}
& G=R^{\top} P-(R-I)^{\top} F \\
& \text { with } G=\left[\begin{array}{c}
b_{1}^{\prime}\left(x_{1}\right)-\frac{\partial c_{1}\left(f_{1}, x_{1}\right)}{\partial x_{1}} \\
b_{2}^{\prime}\left(x_{2}\right)-\frac{\partial c_{2}\left(f_{2}, x_{2}\right)}{\partial x_{2}} \\
\vdots \\
b_{n}^{\prime}\left(x_{n}\right)-\frac{\partial c_{n}\left(f_{n}, x_{n}\right)}{\partial x_{n}}
\end{array}\right], P=\left[\begin{array}{c}
\frac{p_{1}}{\lambda} \\
\frac{p_{2}}{\lambda} \\
\vdots \\
\frac{p_{n}}{\lambda}
\end{array}\right] \text { and } F=\left[\begin{array}{c}
\frac{\partial c_{1}\left(f_{1}, x_{1}\right)}{\partial f_{1}} \\
\frac{\partial c_{2}\left(f_{2}, x_{2}\right)}{\partial f_{2}} \\
\vdots \\
\frac{\partial c_{n}\left(f_{n}, x_{n}\right)}{\partial f_{n}}
\end{array}\right] \text {, }
\end{aligned}
$$

and $t^{*}$ is given by $t_{i}^{*}=\alpha_{i} \sum_{j=1}^{n}\left[b_{j}\left(x_{j}^{*}\right)-c_{j}\left(f_{j}^{*}, x_{j}^{*}\right)-d_{j}\right]-\left[b_{i}\left(x_{i}^{*}\right)-c_{i}\left(f_{i}^{*}, x_{i}^{*}\right)-d_{i}\right], i=$ $1, \ldots, n$.

Note that the matrices $R$ and $R-I$ defining the constraints in (5) reappear in (6), which relates the river geography directly to the ANBS. We can distinguish the effects of resource scarcity $(P)$ from the effects of inflows on the cost of extraction $(F)$.

Theorem 4.1 shows that the monetary transfer paid or received by agent $i$ depends on his bargaining weight $\alpha_{i}$ of the aggregate net surplus $\sum_{j=1}^{n}\left(b_{j}\left(x_{j}^{*}\right)-c_{j}\left(f_{j}^{*}, x_{j}^{*}\right)-d_{j}\right)$ from cooperation minus his own improvement from cooperation $b_{i}\left(x_{i}^{*}\right)-c_{i}\left(f_{i}^{*}, x_{i}^{*}\right)-d_{i}$. Clearly his transfer is increasing in his bargaining weight, i.e., agent $i$ pays less or receives more if he is assigned a larger bargaining weight since $\sum_{j=1}^{n}\left(b_{j}\left(x_{j}^{*}\right)-c_{j}\left(f_{j}^{*}, x_{j}^{*}\right)-d_{j}\right)>0$.

Next we turn to Equation (6). For agent $i, b_{i}^{\prime}$ and $\frac{\partial c_{i}}{\partial x_{i}}$ are the marginal benefit of water use and the marginal cost of water extraction, respectively. Hence, $b_{i}^{\prime}-\frac{\partial c_{i}}{\partial x_{i}}$ is his marginal net benefit of water extraction. Noticing that $R_{j i}=1$ if and only if $j \in S^{i} \cup\{i\}$, it follows that the $i$-th row of System (6) can be written as

$$
b_{i}^{\prime}-\frac{\partial c_{i}}{\partial x_{i}}=\sum_{j=1}^{n} R_{j i} \frac{p_{j}}{\lambda}+\sum_{j=1, j \neq i}^{n}-R_{j i} \frac{\partial c_{j}}{\partial f_{j}}=\sum_{j \in S^{i} \cup\{i\}} \frac{p_{j}}{\lambda}+\sum_{j \in S^{i}}-\frac{\partial c_{j}}{\partial f_{j}}
$$

\footnotetext{
${ }^{4}$ For convenience, we ignore all individual rationality constraints $b_{i}\left(x_{i}\right)-c_{i}\left(f_{i}, x_{i}\right)+t_{i} \geq d_{i}$ because these will be nonbinding, but we do take these into account in the decomposition of the next subsection.

${ }^{5}$ In accordance with taking for granted a unique maximizer to (1), we also take it for granted that (5) has a unique maximizer for the ease of discussion.
} 


$$
=\sum_{j \in S^{i} \cup\{i\}} \frac{p_{j}}{\lambda}+\sum_{j \in S^{i}} \frac{\partial c_{j}}{\partial f_{j}} \frac{\partial f_{j}}{\partial x_{i}},
$$

where the last equality comes from the fact that $f_{j}=e_{j}+\sum_{i \in P^{j}}\left(e_{i}-x_{i}\right)$ and thus $\frac{\partial f_{j}}{\partial x_{i}}=-1$ for $j \in S^{i}$.

First notice from Equation (7) that the marginal net benefit of agent $i$ in the optimum is independent of the bargaining weights $\alpha$. Next notice that the first term of the right-hand side of (7) shows the impact that agent $i$ imposes upon the physical availability of water for his successors. It reflects the resource scarcity of water for agent $i$ and all his successors through the shadow prices $p$ of the the local resource constraints $R x \leq R e$, where $p_{j}>0$ if $x_{j} \leq f_{j}$ is binding, $j \in N$. For agent $i$ this term drops out if $x_{j}^{*}<f_{j}^{*}$ for all $j \in S^{i} \cup\{i\}$, i.e., neither the constraint of $i$ nor any of his successors' constraint is binding. If $x_{j}^{*}=f_{j}^{*}$ for some $j \in S^{i} \cup\{i\}$, then $p_{j}$ decreases when more local water resource $e_{j}$ becomes available. When $p_{k}=0$ for all $k=i$ and all agents $k$ between $i$ and $j$ (and thus all the corresponding constraints are not binding), then the marginal net benefits of agent $i$, agent $j$ and all agents between them will decrease because all these agents could consume some more water when more local water resource $e_{j}$ becomes available. On the other hand, when $p_{k}>0$ for $k=i$ or some agent $k$ between $i$ and $j$, then a higher local resource $e_{j}$ does not allow agent $i$ to consume more water and so the marginal net benefit of $i$ does not change. More water inflow $e_{j}$ and thus a lower $p_{j}$ then induces a higher price $p_{k}$ for agent $k=i$ or at least one other agent $k$ between $i$ and $j$. In this case more local inflow at $j$ induces a higher shadow price, so relatively more scarcity, for at least one agent upstream of $j$.

The second term of the right-hand side of (7) is the sum of all externalities that agent $i$ imposes upon the costs of extraction of all his successors. By assumption $\frac{\partial c_{j}}{\partial f_{j}}<0$ and thus $\frac{\partial c_{j}}{\partial f_{j}} \frac{\partial f_{j}}{\partial x_{i}}>0$ for every successor $j$ of $i$. So, the negative externalities on the extraction costs of his successors lead to higher marginal net benefit for agent $i$. Hence, the consumption $x_{i}^{*}$ in the optimum is lower than what $i$ would like to consume when he is maximizing his own net benefit.

Since every individual term in the summation of the second right-hand term of Equation (7) is strictly positive and $S^{j} \subset S^{i}$ if $j \in S^{i}$, the next proposition holds, showing that the marginal net benefits are decreasing from upstream to downstream.

Proposition 4.2. In the ANBS water allocation $x^{*}$ of the river sharing problem $(N, R, u, e)$, for every $i \in N$ and $j \in S^{i}$ it holds that $b_{i}^{\prime}-\frac{\partial c_{i}}{\partial x_{i}}>b_{j}^{\prime}-\frac{\partial c_{j}}{\partial x_{j}}$.

The intuition is that, the closer agent $i$ is located to one of the sources of the river, the more downstream successors experience such negative externalities from using an extra drop of water by agent $i$. Only the most downstream agent does not induce these externalities. Similarly, if agent $i$ experiences water scarcity, i.e., $p_{i}>0$, then all of his predecessors also experience water scarcity and this positive shadow price $p_{i}$ will show up in their right-hand side of (7). This implies that the closer agent $i$ is to the most-downstream location, he 
will have larger sets of predecessors and this agent's water scarcity is felt by more upstream agents.

Defining $b_{i}^{s}=b_{i}^{\prime}-\frac{\partial c_{i}}{\partial x_{i}}-\sum_{j \in S^{i}} \frac{\partial c_{j}}{\partial f_{j}} \frac{\partial f_{j}}{\partial x_{i}}$ as the societal marginal net benefit of agent $i$, i.e., his own marginal net benefit minus the impact of $x_{i}$ on the marginal extraction costs of his successors, we obtain by rearranging (7) that

$$
b_{i}^{s}=\sum_{j \in S^{i} \cup\{i\}} \frac{p_{j}}{\lambda}, \quad i \in N .
$$

It follows immediately that the societal marginal net benefits are nonincreasing from upstream to downstream and they are all equal to each other if and only if $p_{i}=0$ for all $i<n$.

Corollary 4.3. In the ANBS water allocation $x^{*}$ of the river sharing problem $(N, R, u, e)$, for every $i \in N$ and $j \in S^{i}$ it holds that $b_{i}^{s} \geq b_{j}^{s}$ with at least one strict inequality if and only if $p_{j}>0$ for some $j \neq n$.

The above results generalize the results in Kilgour and Dinar (2001) and Ambec and Sprumont (2002) for the linear river sharing problem to general river geographies captured by $R$ and externalities on the cost of extraction. They observe, as stated in Ambec and Sprumont (2002), that "the marginal benefits decrease (weakly) as one moves downstream and, if two agents have different marginal profits, some constraint must be binding between them." Corollary 4.3 shows the same result for the societal marginal net benefits, which include the marginal own extraction costs and the negative marginal externality costs of extraction on the successors of an agent.

Finally we remark that the ANBS $x^{*}$ and $f^{*}$ can not be implemented through a water market with a uniform water price. What is needed is an institution that can set the correct agent-dependent prices.

\subsection{Decomposition of the computation of the ANBS}

In this subsection, we decompose the ANBS into two separate subproblems of which one has a closed-form solution. The first subproblem immediately arises from the following result. The water uses and inflows of the ANBS coincide with the utilitarian welfare maximizing water uses and inflows. ${ }^{6}$

Theorem 4.4. Let $\left(x^{*}, f^{*}, t^{*}\right)$ be the ANBS for the river sharing problem $(N, R, u, e)$. Then, $x^{*}=x^{U W}, f^{*}=f^{U W}$.

The theorem implies that the aggregate net surplus $\sum_{i=1}^{n}\left[b_{i}\left(x_{i}^{*}\right)-c_{i}\left(f_{i}^{*}, x_{i}^{*}\right)-d_{i}\right]$ at the ANBS is equal to the aggregate net surplus $w-\sum_{i=1}^{n} d_{i}$ resulting from maximizing the

\footnotetext{
${ }^{6}$ When (1) respectively (5) has multiple maximizers, then the result is that $\left(x^{U W}, f^{U W}\right)$ is a maximizer of (1) if and only of there is a maximizer $\left(x^{*}, f^{*}, t^{*}\right)$ of $(5)$ such that $x^{*}=x^{U W}$ and $f^{*}=f^{U W}$.
} 
utilitarian welfare. The intuition of this result is rather straightforward. One of the axioms of the ANBS requires Pareto efficiency and quasi-linear utility functions induce the utility possibility set $U=\left\{u \in \mathbb{R}^{n} \mid \sum_{i=1}^{n} u_{i} \leq w\right\}$. So, Pareto efficiency implies aggregate utilitarian welfare $\sum_{i=1}^{n}\left[b_{i}\left(x_{i}^{*}\right)-c_{i}\left(f_{i}^{*}, x_{i}^{*}\right)\right] \geq w$ and $\left(b_{1}\left(x_{1}^{*}\right)-c_{1}\left(f_{1}^{*}, x_{1}^{*}\right), \ldots, b_{n}\left(x_{n}^{*}\right)-c_{n}\left(f_{n}^{*}, x_{n}^{*}\right)\right) \in U$ implies the opposite weak inequality.

Theorem 4.4 can be related to the discussion on the Coase Theorem. The most wellknown version states that, in the absence of transaction costs, Pareto efficiency arises independent of the assignment of property rights. Note that, in terms of axiomatic solutions, the Coase Theorem states a condition under which the efficiency axiom underlying the ANBS is justified and this axiom is always stated independently of the disagreement points. Hence, given how property rights are translated into disagreement points, the efficiency axiom underlying the ANBS is trivially independent of property rights. More interesting is that the Pareto efficient allocation of water at the ANBS is also independent of the disagreement point and, thus, independent of property rights. Given that we take uniqueness of $x^{U W}$ and $f^{U W}$ for granted, we also obtain the invariance version of the Coase Theorem: in the absence of transaction costs, the same physical allocation arises through negotiations independent of the assignment of property rights. ${ }^{7}$

Since the maximization of utilitarian welfare already characterizes the Pareto efficient water uses $x^{*}$ and inflows $f^{*}$, the next issue is to determine the transfers that maximize the Nash product given $x^{*}$ and $f^{*}$. We stress once more that unanimity requires that each agent must obtain at least his disagreement utility, because otherwise agents who get less will deviate. Without proposing a formal procedure, within our simple framework this can be thought of as follows. As is common in international negotiations over treaties, the negotiations result has to be ratified afterwards by all the participants in the negotiations. If agent $i$ 's utility $u_{i}$ from the treaty is lower than his disagreement utility $d_{i}$, this agent will not ratify and this will prevent the treaty from being implemented. Ratifying any treaty that will give an utility at least equal to the disagreement utility and rejecting otherwise, i.e., ratify if and only if $u_{i} \geq d_{i}$, is a Nash equilibrium strategy of this ratification process for every agent $i \in N$. This argument limits the set $U$ of feasible utility vectors to the bargaining set $U^{I R}(d)=\left\{u \in U \mid u_{i} \geq d_{i}, i \in N\right\}$ of all feasible vectors satisfying individual rationality. Given $x^{*}$ and $f^{*}$, the utility of agent $i$ is given by $u_{i}\left(f_{i}^{*}, x_{i}^{*}, t_{i}\right)=b_{i}\left(x_{i}^{*}\right)-c_{i}\left(f_{i}^{*}, x_{i}^{*}\right)+t_{i}$, which we will write more conveniently as $u_{i}\left(f_{i}^{*}, x_{i}^{*}, t_{i}\right)=u_{i}\left(f_{i}^{*}, x_{i}^{*}, 0\right)+t_{i}$. Successively, we consider the following maximization problem with respect to the monetary transfers

$$
\left.\begin{array}{ll}
\max _{t \in \mathbb{R}^{n}} & \prod_{i=1}^{n}\left(u_{i}\left(f_{i}^{*}, x_{i}^{*}, 0\right)+t_{i}-d_{i}\right)^{\alpha_{i}}, \\
\text { s.t. } & \sum_{i \in N} t_{i} \leq 0, \text { and } u_{i}\left(f_{i}^{*}, x_{i}^{*}, 0\right)+t_{i} \geq d_{i}, i \in N .
\end{array}\right\} .
$$

We have the following result.

\footnotetext{
${ }^{7}$ When (1) respectively (5) has multiple maximizers, then the invariance version of the Coase Theorem can be reformulated in terms of a set of maximizers that is independent of property rights.
} 
Theorem 4.5. Let $\hat{t}$ be the solution of the maximization problem (9). Then, $\left(x^{*}, f^{*}, \hat{t}\right)$ coincides with the ANBS $\left(x^{*}, f^{*}, t^{*}\right)$ for the river sharing problem $(N, R, u, e)$. Moreover,

$$
\hat{t}_{i}=d_{i}+\alpha_{i}\left(w-\sum_{j=1}^{n} d_{j}\right)-u_{i}\left(f_{i}^{*}, x_{i}^{*}, 0\right), i=1, \ldots, n .
$$

From Theorems 4.4 and 4.5, it follows that the computation of the ANBS can be decomposed into two steps: in Step 1, we find the unique maximizer of (1) and, then, we may set $x^{*}=x^{U W}$ and $f^{*}=f^{U W}$. In Step 2, we determine $\hat{t}$, for which we have a closed-form solution given the Pareto efficient $x^{*}$ and $f^{*}$ of Step 1 . Note that we rewrote the transfer when compared to Theorem 4.1. Agent $i$ 's utility in the ANBS is given by $d_{i}+\alpha_{i}\left(w-\sum_{j=1}^{n} d_{j}\right)$ and it is equal to the utility $u_{i}\left(f_{i}^{*}, x_{i}^{*}, 0\right)$ obtained from the use of water plus the monetary transfer $\hat{t}_{i}$. Since the welfare $w$ is larger than $\sum_{j=1}^{n} d_{j}$, the monetary transfer of agent $i$ is increasing in his bargaining weight $\alpha_{i}$. In terms of our previous discussion of the Coase Theorem, the assignment of property rights does have welfare consequences for the agents through the disagreement point, because each agent's utility at the ANBS depends upon the disagreement point and the associated net surplus. Also the financial transfers depend upon the disagreement point. Finally, a central agency with the authority to enforce agreements and who assigns weights among the agents can implement the ANBS in terms of water uses, inflows and monetary transfers. Then, the objective of this agency is equivalent to what the Nash product maximizes in the ANBS.

\subsection{The political economy of property rights}

In this subsection, we specify the disagreement utilities $d \in \mathbb{R}^{n}$ according to the different legal principles of ATS and strict UTI and we investigate and compare the resulting ANBS as obtained in the previous subsection. These legal principles implicitly assign property rights among the agents and an agent may invoke the legal principle that serves his best interest.

Recall from Section 3.2 that $d_{i}^{A T S} \geq 0=d_{i}^{U T I}$ for $i=1 \ldots, n-1$ and that $d_{n}^{A T S}<d_{n}^{U T I}$. For explanatory simplicity we assume in this subsection that $d_{i}^{A T S}>0$ for all $i<n$, so for all agents except agent $n$, the ATS disagreement utility is strictly higher than the strict UTI disagreement utility, only for agent $n$ the opposite holds. However, rational agents are forward looking and are not interested in the disagreement utilities as such, but rather how these affect their final utility in the outcome of the negotiations.

By Proposition 3.1 and 3.2, the disagreement points $d^{A T S}$ and $d^{U T I}$ both belong to the utility possibility set $U$, but the bargaining sets $U^{I R}\left(d^{A T S}\right)$ and $U^{I R}\left(d^{U T I}\right)$ of individual rational utilities differ. According to Theorem 4.5, the final utilities under ATS, respectively strict UTI, become

$$
u_{i}^{A T S}=d_{i}^{A T S}+\alpha_{i}\left(w-\sum_{j=1}^{n} d_{j}^{A T S}\right), i=1, \ldots, n, \text { and }
$$




$$
u_{i}^{U T I}=\alpha_{i}\left(w-d_{n}^{U T I}\right), i=1, \ldots, n-1, \quad u_{n}^{U T I}=d_{n}^{U T I}+\alpha_{n}\left(w-d_{n}^{U T I}\right) .
$$

From the formulas we see two opposite effects that can be related to the disagreement point and the net surplus. As is well-known from bargaining theory, an increase in agent $i$ 's disagreement utility improves this agent's bargaining position in the negotiations and, keeping the other disagreement utilities fixed, will result in an increase in his final utility. However, a change in legal principles shifts other disagreement utilities as well and this results in a different net surplus. The net surplus under UTI is larger than the net surplus under ATS whenever $d_{n}^{U T I}<\sum_{j=1}^{n} d_{j}^{A T S}$, or $d_{n}^{U T I}-d_{n}^{A T S}<\sum_{j=1}^{n-1} d_{j}^{A T S}$. The latter inequality means that the aggregate loss of disagreement utility for agents $i=1, \ldots, n-1$ is larger than the gain in disagreement utility $d_{n}^{U T I}-d_{n}^{A T S}$ for agent $n$. A lower net surplus means that the proportional gains from agreement are also lower. The total effect of these two opposite effects is ambiguous. We have the following result.

Theorem 4.6. Agent $n$ strictly prefers UTI to ATS. If $d_{n}^{U T I}<\sum_{d^{A T S}}^{n} d_{j}^{A T S}$, then agent $i$, $i<n$, weakly prefers ATS to strict UTI if and only if $\alpha_{i} \leq \frac{d_{i}^{A T S}}{\sum_{j=1}^{n} d_{j}^{A T S}-d_{n}^{U T I}}$.

This result states that the most-downstream agent always prefers the strict UTI principle. The intuition is straightforward, this agent becomes the sole owner of all the water and if other agents want to use water they have to pay agent $n$. For the other agents, the answer depends upon their bargaining weights. If agent $i, i<n$, is relatively weak in bargaining, which is reflected in a low bargaining weight, then this agent prefers the ATS principle as the principle defining initial property rights. If agent $i$ is a relatively strong bargainer, he might prefer the strict UTI principle knowing that his bargaining weight can compensate for his lower disagreement utility under strict UTI. Because agent $n$ obtains a larger utility under strict UTI when compared to ATS and the aggregate maximal welfare is $w$, there is at least one agent $i(\neq n)$ who has to get a lower final utility under strict UTI. For an international river that is shared by two countries, downstream prefers UTI and, consequently, upstream prefers ATS. For international rivers involving more countries, it is an empirical research question whether except country $n$, also other countries are better off under strict UTI than under ATS and if so, which countries.

\section{The asymmetric Nash rationing solution}

The individual aspiration levels when all agents invoke UTI, as defined in Section 3.2.2, lie above the Pareto frontier of the utility possibility set, hence these levels are infeasible and cannot be achieved. In this case, we treat the individual aspiration levels as a reference point in which a consensus among the agents requires each agent to bear some losses with respect to his aspiration level. The question then becomes on what compromise outcome the agents agree. 
Many compromise solutions exist in the literature. In this section, we focus on Mariotti and Villar (2005), who study the problem of allocating utility losses among $n$ agents, called the Nash rationing problem, which can be regarded as the translation of the Nash bargaining problem to a situation of compromising on utility losses. The Nash rationing solution is a setvalued solution and consists of the set of points that maximizes a weighted sum of utilities, in which weights are endogenously chosen so that all agents' weighted losses are equal. For problems with transferable utility, the solution is unique and coincides with the unique maximizer of the Nash rationing product. Translated into the river sharing problem, this product is defined as $\prod_{i=1}^{n}\left(d_{i}^{A S P}-u_{i}\right)$ and the Nash rationing solution is the unique maximizer of this product over the set of utility vectors $u \in \mathbb{R}^{n}$ under the constraints $\sum_{j=1}^{n} u_{j} \geq w$ and $u_{i} \leq d_{i}^{A S P}, i=1, \ldots, n$. In this section we propose an asymmetric version of their approach. ${ }^{8}$ We impose the weights exogenously, and the interpretation of these weights is postponed. These weights are given by a vector $\rho=\left(\rho_{1}, \ldots, \rho_{n}\right)$, where $\rho_{i} \geq 0$ and $\sum_{i \in N} \rho_{i}=1$.

Given a weight vector $\rho$, we define the asymmetric Nash rationing solution (ANRS), as the solution of the maximization problem

$$
\max _{\left(u_{1}, \ldots, u_{n}\right)} \prod_{i=1}^{n}\left(d_{i}^{A S P}-u_{i}\right)^{\rho_{i}} \text {, s.t. } \sum_{j=1}^{n} u_{j} \geq w, \text { and } u_{i} \leq d_{i}^{A S P}, \quad i \in N \text {. }
$$

This convex program admits a unique maximizer, denoted $u^{N R S}$. Note that $u_{i}^{N R S}$ is the utility level that each agent gets from the river sharing problem including the monetary transfer. Similar as before for the ANBS, agent $i$ 's monetary transfer closes the gap between his direct net benefit from water use $u_{i}\left(x_{i}^{*}, f_{i}^{*}, 0\right)$ and his final utility $u_{i}^{N R S}$. His transfer is given by $t_{i}^{N R S}=u_{i}^{N R S}-u_{i}\left(x_{i}^{*}, f_{i}^{*}, 0\right)$. Similar to Theorem 4.1, we obtain

$$
u_{i}^{N R S}=d_{i}^{A S P}+\rho_{i}\left(w-\sum_{j=1}^{n} d_{j}^{A S P}\right), \quad i \in N
$$

Since according to Proposition 3.3, $w-\sum_{j=1}^{n} d_{j}^{A S P}<0$, each agent gets a utility level below his individual aspiration level. Moreover, an agent's final utility is decreasing in the weight of the agent. Clearly, an interpretation of $\rho$ in terms of bargaining weights makes no sense, because then a higher bargaining weight implies the counter-intuitive result that this agents gets less utility. Instead, the weight of an agent represents the responsibility of this agent, namely the more weight we put on agent $i$, the more responsibility this agent has to take in making sacrifices to reach a compromise. More responsibility results in a lower utility for an agent. Because the utility is decreasing in the weight, it might even happen that the utility

\footnotetext{
${ }^{8}$ We do not provide an axiomatization, but given the axiomatization results for the ANBS, e.g., Kalai (1977), Kaneko (1980) and Herrero (1989); the convergence results to asymmetric bargaining solutions in strategic bargaining in Herrero (1989); and the axiomatization of the symmetric Nash rationing solution in Mariotti and Villar (2005), it is reasonable to conjecture that the asymmetric version can also be axiomatized. Personal communication with professor Mariotti confirmed this conjecture.
} 
in the ANRS falls below the zero utility that an agent can guarantee himself by blocking agreement and inaction of refraining from using water. The following example illustrates this.

Example 5.1. Consider the case that the tributaries originating at location 1 and 2 merge before location 3 . The agents' responsibility weights are given by $\rho=\left(\frac{3}{5}, \frac{1}{5}, \frac{1}{5}\right)$. The benefit functions, cost functions and water resources are given by,

$$
\begin{aligned}
& b_{i}\left(x_{i}\right)=\sqrt{x_{i}}, c_{i}\left(x_{i}\right)=x_{i}^{2}, \text { and } e_{i}=1, \text { for } i=1,2, \\
& b_{3}\left(x_{3}\right)=16 \sqrt{x_{3}}, c_{3}\left(f_{3}, x_{3}\right)=x_{3}^{2} / f_{3} \text { and } e_{3}=0 .
\end{aligned}
$$

The maximum utilitarian welfare is $w=20.7341$ and by application of (4), we obtain $d^{A S P}=(0.4725,0.4725,20.6274)^{\top}$. Then, formula (12) applied to agent 1 implies $u_{1}^{N R S}=$ $0.4725+0.6(20.7341-20.6274-0.4725-0.4725)=-0.0305<0$.

Given that the utility of inaction is 0 , the question is whether an agent who has to compromise on a negative utility according to (12) is willing to accept the agreement. Without his consent, the agreement fails unanimity. In terms of the ratification process of international treaties mentioned in Section 4.2, ratifying any treaty that will give an utility of at least equal to zero and rejecting otherwise, is a Nash equilibrium strategy of the ratification process for every agent $i \in N$. Therefore, it is natural and, as our example made clear, necessary to impose the participation constraint $u_{i} \geq 0$ for every $i \in N$ in the maximization problem to find the Nash rationing solution. Adding the nonnegativity constraints to the Nash rationing solution complicates the maximization problem, however similar results obtain only that now we have a boundary solution. For the maximization problem including the participation constraints $u_{i} \geq 0, i \in N$, let $T \subset N$ be the set of agents $j \in N$ that receive a utility $u_{j}^{N R S}>0$ at the ANBS. Then, without going into details, we obtain for the Nash rationing solution with nonnegativity constraints that $u_{i}^{N R S}=0$ if $i \in N \backslash T$, and

$$
u_{j}^{N R S}=d_{j}^{A S P}+\frac{\rho_{j}}{\sum_{k \in T} \rho_{k}}\left(w-\sum_{k \in T} d_{k}^{A S P}\right), \text { if } j \in T .
$$

So, the agents in $T$ split the deficit with respect to the total aspiration utilities of the agents in $T$ according to their relative weights within this group. For $T=N$, Equation (13) coincides with (12).

\section{Two numerical examples}

In this section, we provide two numerical examples to illustrate the ANBS under different legal principles regarding the disagreement point in the International Water Law. 


\subsection{Example 1: two agents}

Suppose that only two agents are positioned along the river with $e_{1}=1, e_{2}=0$ and the benefit functions and cost functions given by

$$
\begin{aligned}
& b_{1}\left(x_{1}\right)=\frac{1}{4} \sqrt{x_{1}}, c_{1}\left(x_{1}\right)=\frac{1}{16} x_{1}^{2}, \text { and } \\
& b_{2}\left(x_{2}\right)=\frac{1}{2} \sqrt{x_{2}}, c_{2}\left(f_{2}, x_{2}\right)=\frac{x_{2}^{2}}{f_{2}}, \text { where } f_{2}=1-x_{1} .
\end{aligned}
$$

By application of Theorem 4.4, the maximal utilitarian welfare $w=0.3102$ is attained at $x_{1}^{*}=0.55$ and $x_{2}^{*}=0.15$ with associated utilities $u_{1}^{*}=0.1665$ and $u_{2}^{*}=0.1437$. Note that this step does not involve monetary transfers.

Application of Equations (10) and (11) requires providing the disagreement points first. Under the ATS principle, application of (2) yields the disagreement point $d^{A T S}=(0.1875,0)^{\top}$. Similarly, under the UTI principle of Section 3.2.1, application of (3) yields the disagreement point $d^{U T I}=(0,0.1875)^{\top}$. Figure 1 illustrates that different disagreement points give different bargaining sets, where the utility for agent 1 (2) is positioned on the horizontal (vertical) axis. In this figure, region $A$ is the bargaining set under the ATS principle. For any pair of bargaining weights, the vector of ANBS utility levels according to (10) end up on the segment $c d$. Region $B$ is the bargaining set under the UTI principle of Section 3.2.1 and the vector of ANBS utility levels specified by (11) end up on the segment $a b$. Independent of the bargaining weights, upstream agent 1 always prefers the ATS principle and downstream agent 2 the strict UTI principle.

In terms of Ambec and Sprumont (2002), the downstream incremental solution satisfies the ATS principle and that no coalition of agents can achieve more than their surplus. For $n=2$, their solution maximizes agent 2's utility while keeping agent 1 at his disagreement utility under ATS, i.e., $u_{1}=d_{1}^{A T S}=0.1875$ and $u_{2}=w-d_{1}^{A T S}=0.1227>d_{2}^{A T S}$. This is point $c$ in Figure 1.

If both agents invoke the UTI principle of Section 3.2.2, application of (4) implies the unattainable aspiration level $d^{A S P}=(0.1875,0.1875)^{\top}$, as Figure 1 illustrates. Given any pair of responsibility weights, each agent's utility level in the ANRS follows from Equation (12). In order to reach agreement, each agent has to bear utility losses to end up on the segment $b c$.

In the above example, when considering the Nash rationing solution of Mariotti and Villar (2005), we end up with the middle point of the segment $b c$ which minimizes the weighted sum of individual losses and weights are chosen so that all individual weighted losses are equal. In this situation, we have equal weights for both agents, i.e., $\left(\frac{1}{2}, \frac{1}{2}\right)$, since one unit increase of the utility level for agent 1 must decrease the utility level of agent 2 by 1 unit as well. 


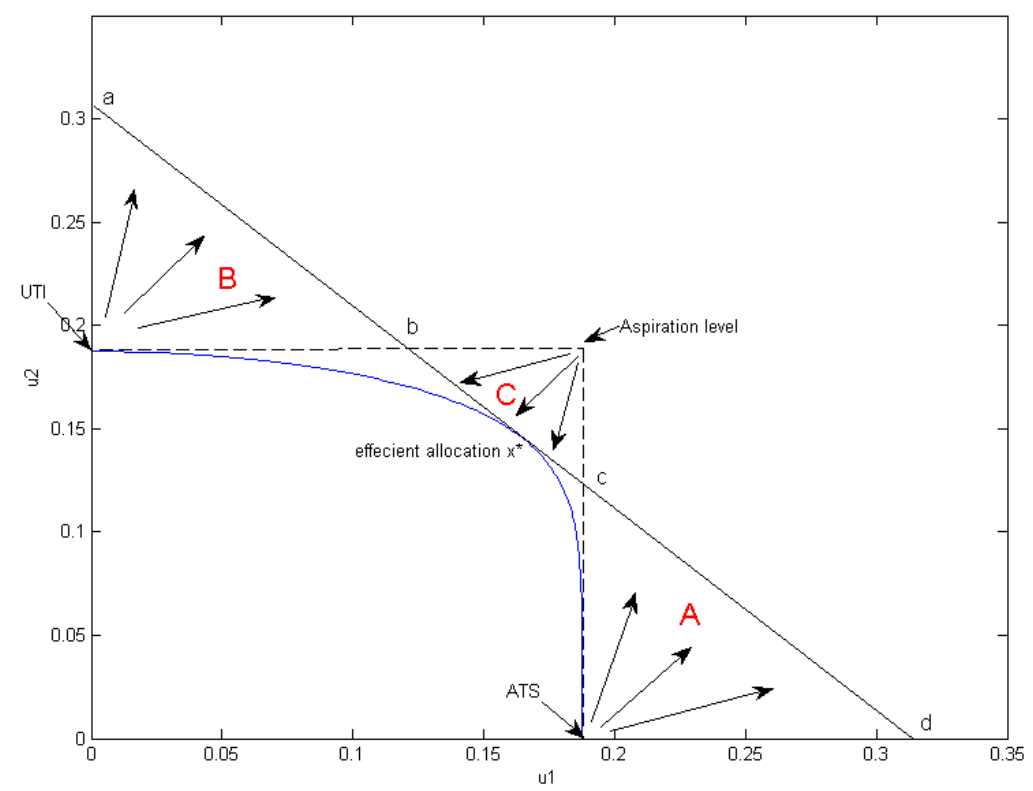

Figure 1: The asymmmetric Nash solutions for the ATS, strict UTI principle and the individual aspiration levels in Example 6.1 (two agents).

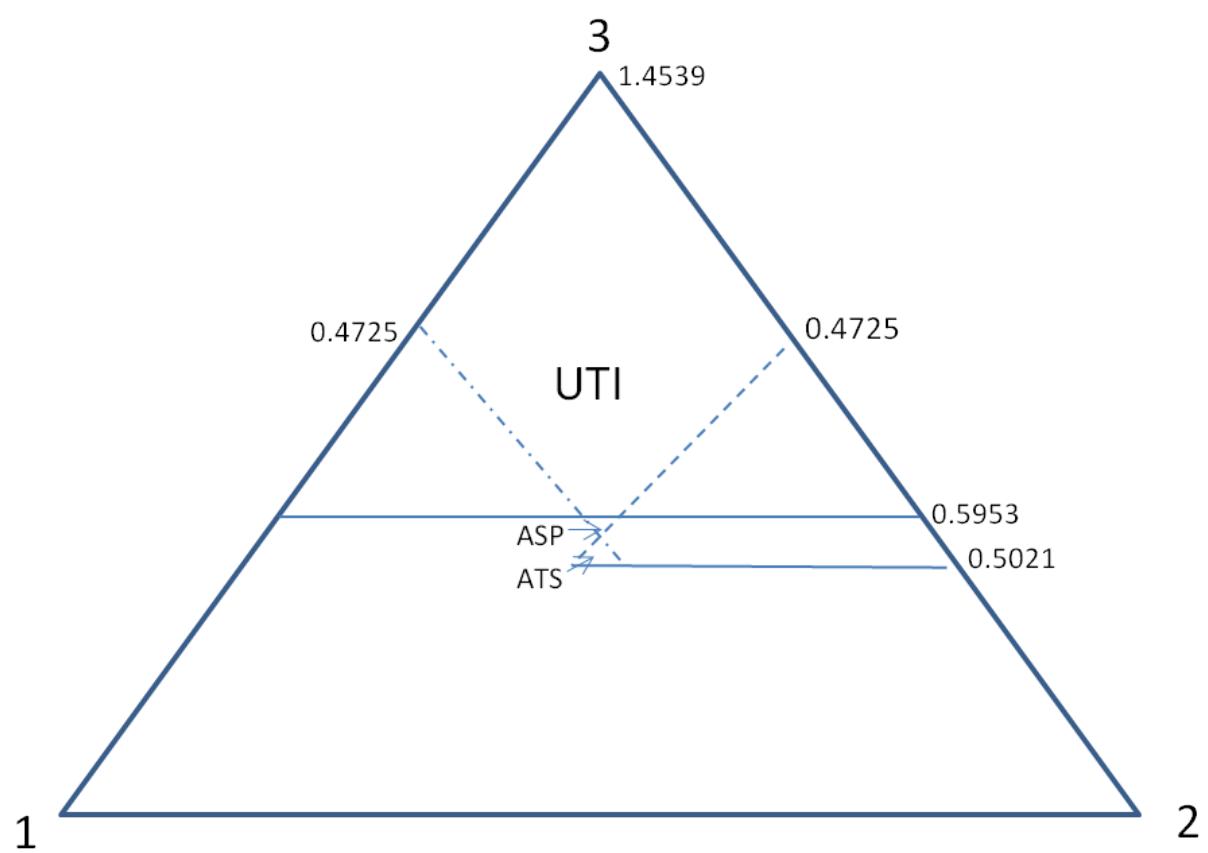

Figure 2: The asymmmetric Nash solutions for the ATS, strict UTI principle and the individual aspiration levels in Example 6.2 (three agents). 
Table 1: Utilities of the agents under ATS, UTI and ASP

\begin{tabular}{|c|c|c|c|}
\hline Legal Principles & Surpluses or Losses & Utility for Agent $i, i=1,2$ & Utility for Agent 3 \\
\hline UTI & 0.8586 & $0+\alpha_{i} 0.8586$ & $0.5953+\alpha_{3} 0.8586$ \\
\hline ATS & 0.0068 & $0.4725+\alpha_{i} 0.0068$ & $0.5021+\alpha_{3} 0.0068$ \\
\hline ASP & 0.0864 & $0.4725-\rho_{i} 10.0864$ & $0.5953-\rho_{3} 0.0864$ \\
\hline
\end{tabular}

\subsection{Example 2: three agents}

We continue with Example 5.1 only that we change the benefit function of agent 3 into $b_{3}\left(x_{3}\right)=\sqrt{x_{3}}$ and that we do not specify the weights. Then, the maximum utilitarian welfare is given by $w=1.4539$. Under ATS, application of (2) yields the disagreement point $d^{A T S}=(0.4725,0.4725,0.5021)^{\top}$. Under strict UTI, the disagreement point is given by $d^{U T I}=(0,0,0.5953)^{\top}$. The vector of individual aspiration levels is given by $d^{A S P}=$ $(0.4725,0.4725,0.5953)^{\top}$. Note that in this situation, we have

$$
d_{i}^{A S P}=d_{i}^{A T S}, \text { for } i=1,2 ; d_{3}^{A S P}=d_{3}^{U T I} .
$$

In Figure 2, we draw the set of possible utility allocations for three agents in the simplex. The small upward-pointing triangular is the bargaining set under ATS, and under individual aspiration levels the bargaining set is the downward-pointing triangular. The large upper triangle, in which agent 3 gets at least a utility of 0.5953 , is the utility bargaining set under strict UTI.

Given the weights, the Nash solution utilities of the agents under the different legal principles are given in Table 1. From this table, we see that agent 3 always prefers strict UTI to ATS, which confirms Theorem 4.6. This can also be deduced from Figure 2, where agent 3's utility in any utility vector in the large upper triangular is larger than this agent's utility in any utility vector in the small upward-pointing triangular. From Table 1, we see that agent 3 prefers strict UTI to ASP. To see this, first recall that $d_{3}^{A S P}=d_{3}^{U T I}$. Then, agent 3 prefers any share of the positive net surplus under strict UTI on top of his disagreement utility under this principle to any compromise under ASP that gives him less than his aspiration level.

Table 1 also implies that agent $i=1,2$ prefers strict UTI to ATS if his bargaining weight $\alpha_{i}>0.5547$, where the lower bound is equal to the threshold stated in Theorem 4.6. Then, agent $i$ can compensate the lower disagreement utility $d_{i}^{U T I}=0$ (when compared to the more favorable $\left.d_{i}^{A T S}=0.4725\right)$ with his share from the larger net surplus $w-d_{3}^{U T I}=0.8586$ (compared to $\left.w-\sum_{i=1,2,3} d_{i}^{A T S}=0.0068\right)$. Table 1 also shows that agents 1 and 2 prefer negotiations under the ATS principle to compromising under the ASP, which is due to $d_{i}^{A S P}=d_{i}^{A T S}$ for $i=1,2$. 


\section{Conclusion}

In this paper, we generalize the river geography and cost functions and investigate several principles from International Water Law. The key finding is that the efficient water allocations are completely determined by the water resources, the river geography and the maximal utilitarian welfare, which can be related to the Coase Theorem. Without monetary compensations there is no room for negotiations. Under ATS and strict UTI, the sum of disagreement utilities is feasible and the agents bargain over the monetary compensations regarding the water transfers with bargaining weights involved. Under the individual aspiration levels, the sum of disagreement utilities is no longer feasible and all agents have to compromise on their utopia levels in order to reach agreement. In this situation, the weights fail an interpretation as bargaining weights and should be interpreted as weights of responsibility in compromising. Higher responsibility weights require larger sacrifices.

The analysis in this paper can be generalized in several directions. Firstly, the ANBS framework is rich enough for further investigation of other principles from International Water Law to investigate how these resolve the river sharing problem. Also alternative bargaining solutions from the literature may be considered. For example, the Kalai-Smorodinsky solution under ATS or strict UTI is already implicitly analyzed in our analysis, because for transferable utility this solution coincides with the symmetric Nash bargaining solution. Secondly, given that every agent may invoke the legal principle that serves him best, a compromise solution over different legal principles may be needed in order to reach agreement. Thirdly, the implementation of the efficient water allocation needs further discussion as well. As argued, the marginal benefit of water use and the marginal cost of water extraction can be interpreted as the consumer price and the producer price, respectively. One potential institutional setup might be that the local producer claims the producer price to the local government. Given the producer price, the local government sets the consumer price according to the efficient allocation scheme. In the framework of multiple agents, we may also need a central agency to regulate local agents to implement the efficient water allocation scheme. Finally, given empirical data, a more ambitious goal is to statistically estimate the bargaining (responsibility) weight in the ANBS (ANRS) from international river treaties, or at the national level, water allocation between provinces. Although river data is often difficult to get, some countries do publish suitable data. This allows us to explore the possibility into

the direction of an econometric estimation of the bargaining (responsibility) weight in the river situation.

\section{References}

Ambec, S. and L. Ehlers (2008). Sharing a river among satiable agents. Games and Economic Behavior 64, 35-50. 
Ambec, S. and Y. Sprumont (2002). Sharing a river. Journal of Economic Theory 10\%, 453-462.

Ansink, E. and H. Houba (2012). Market power in water markets. Journal of Environmental Economics and Management 64, 237-252.

Brink, R. van den, G. van der Laan, and N. Moes (2012). Fair agreements for sharing international rivers with multiple springs and externalities. Journal of Environmental Economics and Management 63, 388-403.

Dinar, A., A. Ratner, and D. Yaron (1992). Evaluating cooperative game theory in water resources. Theory and Decision 32, 1-20.

Giannias, D. and J. Lekakis (1996). Fresh surface water resource allocation between Bulgaria and Greece. Environmental and Resource Economics 8, 473-483.

Herings, P. and A. Predtetchinski (2010). One-dimensional bargaining with Markov recognition probabilities. Journal of Economic Theory 145, 189-215.

Herrero, M. (1989). The Nash program: Non-convex bargaining problems. Journal of Economic Theory 49, 266-277.

Houba, H. (2008). Computing alternating offers and water prices in bilateral river basin management. International Game Theory Review 10, 257-278.

Houba, H., K. P. Do, and X. Zhu (2013). Saving a river: a joint management approach to the Mekong River Basin. Environment and Development Economics 18, 93-109.

Kalai, E. (1977). Nonsymmetric Nash solutions and replication of 2-person bargaining. International Journal of Game Theory 6, 129-133.

Kaneko, M. (1980). An extension of the Nash bargaining solution and the Nash social welfare function. Theory and Decision 12, 135-148.

Kilgour, M. and A. Dinar (2001). Flexible water sharing within an international river basin. Environmental and Resource Economics 18, 43-60.

Laan, G. van der, and N. Moes (2012). Transboundary externalities and property rights: an international river pollution model. Working Paper, Tinbergen Institute, Amsterdam.

Laruelle, A. and F. Valenciano (2008). Noncooperative foundation of bargaining power in committees and the Shapley-Shubik index. Games and Economics Behavior 63, $341-353$.

Locke, J. (1948). In J. W. Cough (Ed.), The Second Treatise of Civil Government. Oxford: Blackwell.

Mariotti, M. and A. Villar (2005). The Nash rationing problem. International Journal of Game Theory 33, 367-377. 
Mas-Colell, A., M. Whinston, and J. Green (1995). Microeconomic theory. Oxford: Oxford University Press.

McCaffrey, S. (1996). The harmon doctrine one hundred years later: buried, not praised. Natural Resources Journal 36, 549-590.

McCaffrey, S. (2001). The Law of International Watercourses. Oxford: Oxford University Press.

Miyakawa, T. (2006). Noncooperative foundation of n-person asymmetric Nash bargaining solution. Working Paper, Osaka University.

\section{Appendix with Proofs}

\section{Proof of Proposition 3.1}

The recursive ATS solution $\left(x_{i}^{A T S}, f_{i}^{A T S}\right)$ satisfies all water resource constraints $x^{A T S} \leq f^{A T S}$ and $f^{A T S}=e+(R-I)\left(e-x^{A T S}\right)$. Therefore, it is a feasible solution of

$$
\max _{x, f \geq 0} \sum_{i=1}^{n}\left(b_{i}\left(x_{i}\right)-c_{i}\left(f_{i}, x_{i}\right)\right) \text { s.t. } R x \leq R e, f=e+(R-I)(e-x) .
$$

Hence, $\sum_{i=1}^{n} d_{i}^{A T S} \leq w$. By Assumption 2.4, the recursively derived local optima $\left(x_{i}^{A T S}, f_{i}^{A T S}\right)$ fail to be the maximizer of (1), because these do not internalize the externalities on the costs of extraction, i.e., $\frac{\partial c_{i}\left(f_{i}, x_{i}\right)}{\partial f_{i}}<0$. Hence, $\sum_{i=1}^{n} d_{i}^{A T S}<w$.

\section{Proof of Proposition 3.3}

By definition of (1), we have

$$
\begin{aligned}
w & =\sum_{i=1}^{n}\left(b_{i}\left(x_{i}^{U W}\right)-c_{i}\left(f_{i}^{U W}, x_{i}^{U W}\right)\right)<\sum_{i=1}^{n}\left(b_{i}\left(x_{i}^{U W}\right)-c_{i}\left(f_{i}^{A S P}, x_{i}^{U W}\right)\right) \\
& \leq \sum_{i=1}^{n}\left(b_{i}\left(x_{i}^{A S P}\right)-c_{i}\left(f_{i}^{A S P}, x_{i}^{A S P}\right)\right)=\sum_{i=1}^{n} d_{i}^{A S P} .
\end{aligned}
$$

The strict inequality comes from the fact that $c_{i}\left(f_{i}, x_{i}\right)$ is decreasing in $f_{i}$ and $f_{i}^{U W}<f_{i}^{A S P}$.

\section{Proof of Proposition 4.2}

Without loss of generality, renumber the agents such that agent $i+1$ is agent $i$ 's downstream neighbor. By the tree structure of the river, $i+1 \in S^{i}=\{i+1\} \cup S^{i+1}$ and combined with 
(7) we obtain

$$
\begin{aligned}
b_{i}^{\prime}-\frac{\partial c_{i}}{\partial x_{i}} & =\sum_{j \in S^{i} \cup\{i\}} \frac{p_{j}}{\lambda}-\sum_{j \in S^{i}} \frac{\partial c_{j}}{\partial f_{j}}=\underbrace{\frac{p_{i}}{\lambda}-\frac{\partial c_{i+1}}{\partial f_{i+1}}}_{>0}+\sum_{j \in S^{i+1} \cup\{i+1\}} \frac{p_{j}}{\lambda}-\sum_{j \in S^{i+1}} \frac{\partial c_{j}}{\partial f_{j}} \\
& >\sum_{j \in S^{i+1} \cup\{i+1\}} \frac{p_{j}}{\lambda}-\sum_{j \in S^{i+1}} \frac{\partial c_{j}}{\partial f_{j}} \stackrel{(7)}{=} b_{i+1}^{\prime}-\frac{\partial c_{i+1}}{\partial x_{i+1}} .
\end{aligned}
$$

Recursive repetition of these arguments implies the stated result.

\section{Proof of Theorem 4.1}

After substitution of $f$, we define $M=\prod_{i=1}^{n}\left(b_{i}\left(x_{i}\right)-c_{i}\left(e_{i}+\sum_{j \in P^{i}}\left(e_{j}-x_{j}\right), x_{i}\right)+t_{i}-d_{i}\right)^{\alpha_{i}}$ for notational convenience. Let $L(x, t, p, \lambda)$ denote the Lagrangian with shadow prices $p$ and $\lambda$ are defined in Theorem 4.1. Then, the first-order conditions for $x^{*}$ and $t^{*}$ read

$$
\begin{aligned}
& \frac{\partial L}{\partial x_{i}}: \alpha_{i} \frac{M}{b_{i}-c_{i}+t_{i}-d_{i}}\left(b_{i}^{\prime}-\frac{\partial c_{i}}{\partial x_{i}}\right)+\sum_{j \in S^{i}} \alpha_{j} \frac{M}{b_{j}-c_{j}+t_{j}-d_{j}} R_{j i} \frac{\partial c_{j}}{\partial f_{j}}-\sum_{j \in S^{i} \cup\{i\}} R_{j i} p_{j}=0, \\
& \frac{\partial L}{\partial t_{i}}: \alpha_{i} \frac{M}{b_{i}-c_{i}+t_{i}-d_{i}}-\lambda=0 .
\end{aligned}
$$

Writing the first $n$ equations into matrix form, we obtain

$$
R^{T} P=G+\left(R^{T}-I\right) F
$$

where $P, G$ and $F$ are stated in Theorem 4.1. With respect to the monetary transfers, we have

$$
\frac{M}{b_{i}-c_{i}+t_{i}-d_{i}} \alpha_{i}=\lambda, i=1, \ldots, n
$$

Dividing the equation for $i=1$ by the one for $i$, we obtain

$$
t_{i}=\frac{\alpha_{i}}{\alpha_{1}}\left(b_{1}-c_{1}-d_{1}\right)+\frac{\alpha_{i}}{\alpha_{1}} t_{1}-\left(b_{i}-c_{i}-d_{i}\right) .
$$

This establishes a relationship between $t_{i}$ and $t_{1}$ for all $i \geq 2$. Substitution of these expressions in $\sum_{i=1}^{n} t_{i}=0$ yields,

$$
t_{1}+\left[\frac{\alpha_{2}}{\alpha_{1}}\left(b_{1}-c_{1}-d_{1}\right)+\frac{\alpha_{2}}{\alpha_{1}} t_{1}-\left(b_{2}-c_{2}-d_{2}\right)\right]+\ldots+\left[\frac{\alpha_{n}}{\alpha_{1}}\left(b_{1}-c_{1}-d_{1}\right)+\frac{\alpha_{n}}{\alpha_{1}} t_{1}-\left(b_{n}-c_{n}-d_{n}\right)\right]=0 .
$$

From which, $t_{1}$ can be solved as,

$$
t_{1}=\alpha_{1} \sum_{j=1}^{n}\left(b_{j}-c_{j}-d_{j}\right)-\left(b_{1}-c_{1}-d_{1}\right) .
$$


Similarly, we obtain

$$
t_{i}=\alpha_{i} \sum_{j=1}^{n}\left(b_{j}-c_{j}-d_{j}\right)-\left(b_{i}-c_{i}-d_{i}\right)
$$

\section{Proof of Theorem 4.4}

It suffices to show that the first-order conditions coincide with those for the ANBS given in the proof of Theorem 4.1. After substitution of $f$, we have to solve

$$
\max _{x} \sum_{i \in N}\left(b_{i}\left(x_{i}\right)-c_{i}\left(e_{i}+\sum_{j \in P^{i}}\left(e_{j}-x_{j}\right), x_{i}\right)\right) \text {, s.t. } R x \leq R e(\tilde{p}) .
$$

where $\tilde{p}$ is the vector of Lagrange multipliers. The Lagrangian is given by

$$
\tilde{L}(x, \tilde{p})=\sum_{i \in N}\left(b_{i}\left(x_{i}\right)-c_{i}\left(e_{i}+\sum_{j \in P^{i}}\left(e_{j}-x_{j}\right), x_{i}\right)\right)+\tilde{p}^{\top}(R e-R x)
$$

Taking first-order conditions, for $x_{i}$, we have

$$
b_{i}^{\prime}-\frac{\partial c_{i}}{\partial x_{i}}+\sum_{j=1, j \neq i}^{n} R_{j i} \frac{\partial c_{i}}{\partial f_{i}}-\sum_{j=1}^{n} R_{j i} \tilde{p}_{j}=0 .
$$

We compare these with the first-order conditions for the ANBS

$$
\frac{\partial L}{\partial x_{i}}: \alpha_{i} \frac{M}{b_{i}-c_{i}+t_{i}-d_{i}}\left(b_{i}^{\prime}-\frac{\partial c_{i}}{\partial x_{i}}\right)+\sum_{j=1, j \neq i}^{n}\left(\alpha_{j} \frac{M}{b_{j}-c_{j}+t_{j}-d_{j}}\right) R_{j i} \frac{\partial c_{j}}{\partial f_{j}}-\sum_{j=1}^{n} R_{j i} p_{j}=0 .
$$

Let $\alpha_{i} \frac{M}{b_{i}-c_{i}+t_{i}-d_{i}}=\lambda$. Then, a simple normalization of the Lagrange multipliers $\left(\frac{p_{j}}{\lambda}\right)$ will get the stated result.

\section{Proof of Theorem 4.5}

The Lagrange function for the maximization problem is ${ }^{9}$

$$
L=\prod_{i=1}^{n}\left(u_{i}\left(f_{i}^{*}, x_{i}^{*}, 0\right)+t_{i}-d_{i}\right)^{\alpha_{i}}-\lambda \sum_{i} t_{i} .
$$

The first-order conditions read

$$
\frac{\partial L}{\partial t_{i}}: \alpha_{i} \frac{M}{u_{i}\left(f_{i}^{*}, x_{i}^{*}, 0\right)+t_{i}-d_{i}}-\lambda=0
$$

\footnotetext{
${ }^{9}$ We omit the individual rationality constraint in the Lagrange function. Later on we will check for this.
} 
For $j \neq 1$, we have

$$
t_{j}=\frac{\alpha_{j}}{\alpha_{1}}\left[u_{1}\left(f_{1}^{*}, x_{1}^{*}, 0\right)-d_{1}\right]+\frac{\alpha_{j}}{\alpha_{1}} t_{1}-\left[u_{j}\left(f_{i}^{*}, x_{i}^{*}, 0\right)-d_{j}\right] .
$$

This establishes a relationship between $t_{j}$ and $t_{1}$ for all $j \geq 2$. Substitution of these expression into $\sum_{i=1}^{n} t_{i}=0$ and solve for $t_{1}$, we obtain,

$$
\hat{t}_{1}=\alpha_{1} \sum_{j=1}^{n}\left[u_{j}\left(f_{j}^{*}, x_{j}^{*}, 0\right)-d_{j}\right]-\left[u_{1}\left(f_{1}^{*}, x_{1}^{*}, 0\right)-d_{1}\right] .
$$

It remains to check for the individual rationality constraint. Indeed,

$$
u_{1}\left(f_{1}^{*}, x_{1}^{*}, 0\right)+\hat{t}_{1}=d_{1}+\alpha_{1}\left(w-\sum_{j=1}^{n} d_{j}\right)>d_{1}
$$

since $w>\sum_{j=1}^{n} d_{j}$. Similar results follow for $\hat{t}_{j}$ for all $j \geq 2$. Hence, $\hat{t}$ coincides with $t^{*}$ stated in Theorem 4.1 .

\section{Proof of Theorem 4.6}

For agent $n$, we have

$$
u_{n}^{U T I}>u_{n}^{A T S} \Longleftrightarrow\left(1-\alpha_{n}\right)\left(d_{n}^{U T I}-d_{n}^{A T S}\right)>-\alpha_{n} \sum_{j=1}^{n-1} d_{j}^{A T S} .
$$

The inequality always holds, because the right-hand side is positive and the left-hand side is at most 0 . For agent $i$, we have

$$
u_{i}^{A T S} \leq u_{i}^{U T I} \Longleftrightarrow \alpha_{i} \leq \frac{d_{i}^{A T S}}{\sum_{j=1}^{n} d_{j}^{A T S}-d_{n}^{U T I}} .
$$

Because $d_{n}^{U T I}<\sum_{j=1}^{n} d_{j}^{A T S}$, the $\leq$ is preserved and the right-hand side is nonnegative. 